\title{
The Integrated Detection System of UAV Power
}

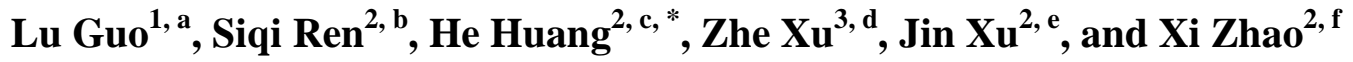 \\ ${ }^{1} \mathrm{Xi}$ ' an ASN Technology Group Company, Xi' an 710065, China \\ ${ }^{2}$ School of Electronic and Control Engineering, Chang'an University, Xi' an, 710064, China \\ ${ }^{3}$ The 20th Research Institute, Xi' an, 710068, China \\ a tolovelulu@126.com, ${ }^{b} 409480920 @ q q . c o m,{ }^{c}$ huanghe@chd.edu.cn, ${ }^{d}$ xuzhe0330@163.com, \\ e405708023@qq.com, ${ }^{\text {e}}$ 1452355278@qq.com.
}

Keywords: UAV, detection, power, performance, test

Abstract: The process of performance testing and parameter debugging of existing UAV is complicated. At the same time, the working environment of UAV is mostly unreachable by human beings, and the flight distance is too far away, causing the UAV to be out of control, which will cause huge property losses. This paper proposes a UAV power comprehensive detection system, which uses the serial port of the computer to debug the performance and system parameters of the UAV. The assistant of serial port debugging includes the system initialization module, the work control module of power system, the mode switching module of power system, the parameter display module of power system, the fault alarm module of power system, and the display module of system time. The coordinated work of multiple parts constitute the integrated detection system of the power system of UAV. After experimental testing, the system realized the monitoring of the internal temperature of the UAV, the monitoring of the speed of the power system, and the adjustment of the fuel and tank pressure. Experiments show that the system can successfully monitor the internal data of the UAV and debug the performance of each module to ensure the stable performance of the UAV.

\section{Introduction}

Nowadays the industry of UAV is developing rapidly, all sorts of UAV have been invented in succesion, The UAV has been used for aerial photography, military, detection, and so on, however, with the increasing demand and requirements of UAV. For example, military UAV used for aerial photography and detection on cliffs or at sea has to undergo complex, strict and comprehensive monitoring before being manufactured and put into use due to their high price, harsh working environment and strict requirements. However, the test process of UAV adopts direct flight test. The risk of damage is extremely high and the cost is huge. The specific parameters are too precise and the actual flight test takes a lot of time. Therefore, using a kind of serial debugging assistant with the upper computer for monitoring and parameter display, upper parameter debugging and data return value display can save a lot of money and manpower, save time, and ensure safety and stability of performance before formal operation. 


\section{Software Architecture of the System}

The system architecture of the integrated detection software for fixed-wing UAV power system is shown in figure 1.

The integrated detection software of the fixed wing UAV power system includes the module of system initialization, the working control module of power system, the module of power system mode switching, the module of power system parameter, the warning module of power system fault and the display module of system time.

The module of system initialization includes serial port initialization, variable initialization, timer initialization and form initialization. The module of power system working control includes fuel control, engine control and pressurization control. The module of power system mode switching includes normal mode, test mode and self - check mode. The parameter integrated display module of power system includes fuel controller parameter display, engine measurement, control unit parameter display and pressure controller parameter display. The warning module of power system fault includes fuel controller fault warning, engine measurement and control unit fault warning, pressure controller fault warning.

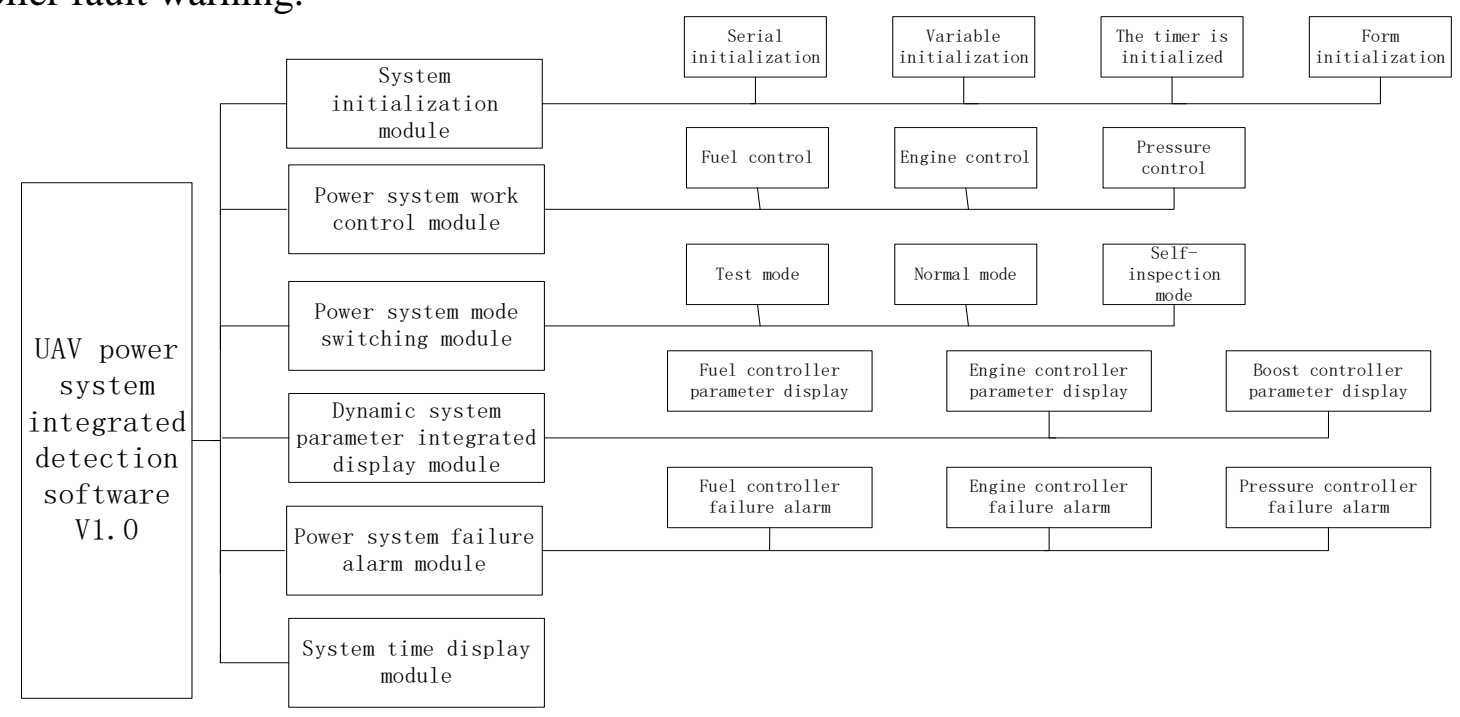

Fig. 1 The software frame of the fixed wing UAV power integrated detection system

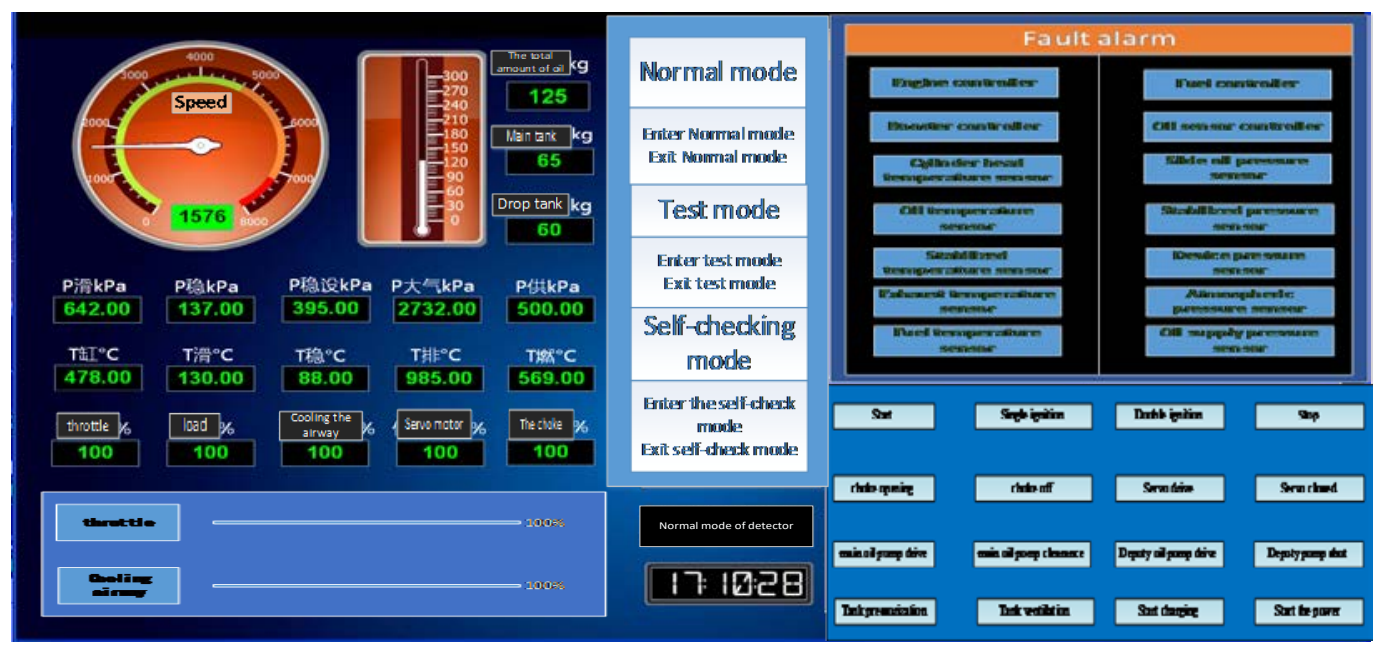

Fig. 2 The software frame of the fixed wing UAV power integrated detection system 
The display module of system time is used to display system time. The Human-computer interaction interface of the integrated detection software for fixed-wing UAV power system is shown in Fig 2.

\subsection{System initialization module}

The system initialization module includes serial port initialization, variable initialization, timer initialization and form initialization. The system is initialized automatically after the software is opened. The serial port adopts RS232 interface, the serial number is COM1, and the initialization is set to baud rate of $19200 \mathrm{bps}$, parity check is odd check, and 1 stop bit. The period of timer initialization is $200 \mathrm{~ms}$. The size of form is $1920 * 1080$, full screen displaying, assigning type and initial value for each variable.

\subsection{Control module of power system work}

The UAV power system includes three parts: engine controller, fuel controller and pressurization controller.The fixed wing UAV power system integrated detection software realizes the control function of the power system through the control module of power system work. This module contains 18 buttons, as shown in figure 2.

The command control button of the power system is used to control the start, ignition mode, stop, damper switch, servo motor switch, starting solenoid valve switch, main oil pump switch and auxiliary oil pump switch of fuel controller, pressure controller switches between the pressurized fuel tank and the air. The command control button of the power system in figure 3 and its corresponding functions are shown in table 1.

Table 1 Power system control command button function

\begin{tabular}{|c|c|c|}
\hline serial number & name of the button & function \\
\hline 1 & starting & engine controller performs the starting process \\
\hline 2 & Single ignition & ignition control box is disconnected \\
\hline 3 & Double ignition & dual-ignition control box is disconnected \\
\hline 4 & parking & engine controller performs the shutdown \\
process
\end{tabular}

The power system adjustment command buttons in figure 4 and their corresponding functions are shown in table 2 . 
Table 2 functions of power system adjustment command button

\begin{tabular}{|c|c|c|c|c|}
\hline $\begin{array}{c}\text { Serial } \\
\text { number }\end{array}$ & Button name & function & Control range & $\begin{array}{c}\text { accurac } \\
\mathrm{y}\end{array}$ \\
\hline 1 & throttle & Adjust the engine throttle position & $0 \% \sim 100 \%$ & $1 \%$ \\
\hline 2 & Cooling airway & $\begin{array}{c}\text { Adjust the engine cooling channel } \\
\text { position }\end{array}$ & $0 \% \sim 100 \%$ & $1 \%$ \\
\hline
\end{tabular}

The opening range of the throttle valve ranges from $0 \%$ to $100 \%$. After "+" is clicked, the opening value of the throttle valve increases by $1 \%$. After "-" is clicked, the opening value of the throttle valve decreases by $1 \%$.

After adjusting to the appropriate position, clicking the "throttle" button is sending the throttle opening control command to the power system controller to adjust the throttle opening to the designated position.

Cooling airway opening ranges from $0 \% \sim 100 \%$. After "+" is clicked, coolant airway opening value increases by $1 \%$. After "-" is clicked, cooling airway opening value reduced by $1 \%$. After adjusting to the appropriate position, clicking the "cooling airway" button is sending the control command of cooling airway opening to the power system controller to adjust the cooling airway opening to the designated position.

\subsection{Switching module of power system mode}

The module of power system modes switching includes normal mode, test mode and self - check mode. The integrated detection software of the dynamic system of fixed-wing UAV can switch three working modes through the mode switch module on the user interface, including normal mode, test mode and self-check mode.

Click "enter normal mode" button, and the detector sends the command to enter normal mode to the power system. The power system switches to normal mode. Under the normal mode, the buttons of "single ignition, double ignition, damper opening, damper closing, servo opening, servo closing, start energizing and start the power cutting" cannot be used, which turns gray. Click the "exiting normal mode" button, and the detector sends the normal mode exiting command to the power system. The power system exits the normal mode, and all the control command buttons of the power system turn color, as it was shown in Fig.2.

But the control command fails, the power system does not receive the control command. Click "enter test mode" button, and the detector sends the entering test mode command to the power system. The power system switches to the test mode. Under the test mode, all the control command buttons of power system are colored, as it was shown in Fig.2.

The control command is effective, and the power system executes the corresponding action after receiving the control command. Click the "exiting test mode" button, and the detector sends the exiting test mode command to the power system. The power system exits the test mode, and the control command buttons of the power system are all colored, as it was shown in Fig.2.

But the control command fails, the power system does not receive the control command. Click the button of "entering self-check mode", and the detector sends the command of entering self-check mode to the power system. The power system switches to self-check mode, and the whole system self-check is conducted automatically. In the self-check mode, the control command buttons of power system cannot be used, all of them are grey. Click "exiting self-test mode", the detector sends the command to the power system to exit the self-test mode, the power system to exit the self-test mode, and the control command buttons of power system are all colored. But the control command fails, the 
power system does not receive the control command.

\subsection{Comprehensive display module of power system parameters}

The engine fault detection software displays the sensor information that sent back by each sensor on the monitor interface in the forms of data and charts, which is convenient for manual judgment or automatic display of fault information, and troubleshooting common engine failures. The information of more than 20 parameters, such as the ignition circuit of the engine, static pressure of the cylinder block, fuel flow and pressure, as they were shown in figure 1.

The engine speed is displayed in real time in the form of instrument panel control and text box. The gauge range of the instrument panel is 0 8000 in RPM. The text box displays the actual value of current speed, which is accurate to 1 revolution. The pointer synchronously indicates the actual value of engine speed. The fuel tank is displayed in real time in the form of flow meter and text box. The range of the flowmeter is $0 \sim 300 \mathrm{in} \mathrm{kg}$, and the total flow text box shows the actual time value of the current total flow of the oil tank, the text box of the main oil tank shows the actual time value of the current flow of the main oil tank, and the text box of the auxiliary oil tank shows the actual time value of the current flow of the auxiliary oil tank, which is accurate to $1 \mathrm{~kg}$. The flowmeter synchronously indicates the actual time value of the total oil amount in the tank. In the figure 1, the power system of lubricating oil pressure, stabilizing pressure pressure and temperature of the cylinder head, air static pressure, oil pressure, oil temperature and stabilizing pressure temperature, the temperature of exhaust gas temperature, fuel temperature, equipment, throttle position, engine load, cooling of the opening of the airway, servo motor position, the choke, stabilizing pressure setting location adopt the form of a text box, according to the text name and corresponding relationship of the dynamic system, as they were shown in table 3.

Table 3 the text name of the control corresponds to the power system parameters

\begin{tabular}{|c|c|c|c|c|}
\hline \multicolumn{5}{|c|}{ Power system parameter display } \\
\hline Serial number & Control name & Display parameter & unit & range \\
\hline 1 & P slip & Sliding oil pressure & $\mathrm{kPa}$ & $0 \sim 65535$ \\
\hline 2 & P stability & Ballast pressure & $\mathrm{kPa}$ & $0 \sim 65535$ \\
\hline 3 & T cylinder & Head temperature & ${ }^{\circ} \mathrm{C}$ & $0 \sim 65535$ \\
\hline 4 & P atmosphere & Atmospheric static pressure & $\mathrm{kPa}$ & $0 \sim 65535$ \\
\hline 5 & P supply & Oil supply pressure & $\mathrm{kPa}$ & $0 \sim 65535$ \\
\hline 6 & T slip & Oil temperature & ${ }^{\circ} \mathrm{C}$ & $0 \sim 65535$ \\
\hline 7 & T stability & Ballast temperature & ${ }^{\circ} \mathrm{C}$ & $0 \sim 65535$ \\
\hline 8 & T row & Exhaust temperature & ${ }^{\circ} \mathrm{C}$ & $0 \sim 65535$ \\
\hline 9 & $\mathrm{~T}$ fire & Fuel temperature & ${ }^{\circ} \mathrm{C}$ & $0 \sim 65535$ \\
\hline 10 & T equipment & Equipment temperature & ${ }^{\circ} \mathrm{C}$ & $0 \sim 65535$ \\
\hline 11 & throttle & Throttle position & $\%$ & $0 \sim 100$ \\
\hline 12 & load & Engine load position & $\%$ & $0 \sim 100$ \\
\hline 13 & $\begin{array}{c}\text { Cooling } \\
\text { airway }\end{array}$ & Cooling airway opening & $\%$ & $0 \sim 100$ \\
\hline 14 & Servo motor & Servo position & $\%$ & $0 \sim 100$ \\
\hline 15 & Choke damper & Choke position & $\%$ & $0 \sim 100$ \\
\hline 16 & P stability & $\begin{array}{c}\text { The stabilizer sets the } \\
\text { position }\end{array}$ & $\mathrm{kPa}$ & $0 \sim 65535$ \\
\hline
\end{tabular}




\subsection{Fault warning module of power system}

The fault warning modules of power system includes fuel controller fault detection, engine controller fault detection and pressurization controller fault detection. Respectively on the engine controller, fuel oil controller, pressure controller, cylinder head temperature sensor, oil temperature sensor and temperature sensor, the stable pressure box, exhaust temperature sensor, fuel temperature sensor, oil amount sensing annunciator, lub oil pressure sensor, voltage regulator pressure sensor, pressure sensor, atmospheric pressure sensor, oil pressure sensor and other 14 key indicators for fault detection. The fault warning modules of the power system automatically display the fault information sent back by the power system on the monitor interface, which is convenient for fault positioning and subsequent manual troubleshooting of common engine failures. As they were shown in table 4 .

Table 4 Faults detection table of power system

\begin{tabular}{|c|c|c|c|}
\hline $\begin{array}{c}\text { Serial } \\
\text { number }\end{array}$ & name & State $\mathbf{1}$ & State 2 \\
\hline 1 & Engine controller & Normal: green & Failure: red \\
\hline 2 & Fuel controller & Normal: green & Failure: red \\
\hline 3 & Booster controller & Normal: green & Failure: red \\
\hline 4 & Cylinder head temperature & Normal: green & Failure: red \\
\hline 5 & Oil temperature sensor & Normal: green & Failure: red \\
\hline 6 & Stabilized temperature sensor & Normal: green & Failure: red \\
\hline 7 & Exhaust temperature sensor & Normal: green & Failure: red \\
\hline 8 & Fuel temperature sensor & Normal: green & Failure: red \\
\hline 9 & Oil sensing signal & Normal: green & Failure: red \\
\hline 10 & Slide oil pressure sensor & Normal: green & Failure: red \\
\hline 11 & Stabilized pressure sensor & Normal: green & Failure: red \\
\hline 12 & Device pressure sensor & Normal: green & Failure: red \\
\hline 13 & Atmospheric pressure sensor & Normal: green & Failure: red \\
\hline 14 & Oil supply pressure sensor & Normal: green & Failure: red \\
\hline
\end{tabular}

Each indicator in table 4 has two situations, and the text normally appears green, as it is shown in Fig. 2.

\section{Test Results}

Through the long time test operation of the system, the comprehensive detection software is stable and reliable under various working modes of the fixed-wing UAV power system. Fixed-wing UAV power system comprehensive testing software can be out of the plane system, an independent testing of power system, on the ground in the face of power system debug, comprehensive system of detecting the function of the power system, performance, security, stability, reliability, accurate fault location, providing favorable evidence for subsequent analysis of failure causes. In the ground test 
stage, problems are found and faults are removed in time, which ensures the safety of UAV flight to a great extent.

\section{Conclusions}

The integrated detection software of fixed wing UAV power system successfully solves the problems of low integration, poor automation, low efficiency and low accuracy in the current manual detection of small and medium-sized UAV power system, and has the advantages of easy operation, easy maintenance and easy expansion. In the process of software designment, the system's design requirements such as multi-parameter detection, highly intelligent, comprehensive, and convenient operability make the designment of testing software particularly important, and its reliability, stability and maintainability are especially critical.

\section{References}

[1] Takumi Matsuda, Toshihiro Maki. Experimental Evaluation of Accuracy and Efficiency of Alternating Landmark Navigation by Multiple AUVs [J]. IEEE Journal of Oceanic Engineering, 2018, 43(2):288 - 310.

[2] Marc Carreras; Juan David Hernández. Sparus II AUV-A Hovering Vehicle for Seabed Inspection [J]. IEEE Journal of Oceanic Engineering, 2018, 43(2): 344-355.

[3] Chen-Wei Chen, Jen-Shiang Kouh. Modeling and Simulation of an AUV Simulator with Guidance System [J]. IEEE Journal of Oceanic Engineering, 2013, 38(2):211-225

[4] Wang X Y,Qin H P. Development of experimental device for sensing signal detection and intelligent instrument integration [J]. Experiment Technology and Management, 2014, 31(3): 66-69.

[5] XIA H M, XIE G X, PENG J H. Research of general test platform for naval navigation equipments[J]. Electronic Design Engineering, 2010, 18(7): 107-112

[6] Ding S B, Xiao C S. Modeling of x-type quadrotor UAV and four-element number control[J], Journal of System Simulation, 2015,27 (12) : 3057-3062.

[7] Lin S, Lu X L, et al. A UAV integrated detection and control system based on virtual instruments [J]. Measurement and Control Technology, 2002, 21 (5) : 53-54 